FORMATION Formation emploi

Revue française de sciences sociales

131 | Juillet-Septembre 2015

Le Bac Pro a 30 ans

\title{
Point de vue : Bachelier ou professionnel ?
}

\section{Claude Grignon}

\section{OpenEdition}

Journals

Édition électronique

URL : http://journals.openedition.org/formationemploi/4516

DOI : 10.4000/formationemploi.4516

ISSN : 2107-0946

\section{Éditeur}

La Documentation française

\section{Édition imprimée}

Date de publication : 15 octobre 2015

Pagination : 163-168

ISSN : 0759-6340

\section{Référence électronique}

Claude Grignon, «Point de vue : Bachelier ou professionnel ? », Formation emploi [En ligne], 131 | JuilletSeptembre 2015, mis en ligne le 15 octobre 2017, consulté le 30 octobre 2020. URL : http:// journals.openedition.org/formationemploi/4516; DOI : https://doi.org/10.4000/formationemploi.4516 


\title{
Point de vue
}

\section{Bachelier ou professionnel ?}

\author{
Claude Grignon \\ Sociologue, directeur de recherche honoraire à I'INRA \\ (Institut national de la recherche agronomique)
}

Le baccalauréat professionnel ne prépare pas à l'exercice des professions, i.e. des professions libérales, comme les professions juridiques ou la médecine, mais, à l'autre extrémité de l'échelle sociale et de la hiérarchie des prestiges, à la pratique de ce que l'on appelle les « métiers ». L'opposition entre métiers et professions recouvre l'opposition entre manuel et non manuel.

Si arbitraires que soient les classements et les qualifications auxquels cette opposition donne lieu, elle n'est pas dépourvue de fondements objectifs, et c'est seulement en examinant les cas où elle s'applique à la réalité que l'on fera pleinement ressortir cet arbitraire. Il faut en effet distinguer entre les savoirs que l'on a en tête et les tours de main, entre ce qui s'enseigne par la parole ou l'écrit et ce qui s'apprend par l'observation, l'imitation, le langage du corps ${ }^{1}$, bref entre l'enseignement et l'apprentissage. Les capacités proprement manuelles, l'habileté, l'adéquation et la précision des gestes et des postures, la virtuosité s'acquièrent par l'exercice en faisant appel à une espèce particulière de mémoire, la mémoire procédurale.

Ces capacités sont aussi nécessaires pour le pianiste, qui doit avoir ses morceaux « dans les doigts " pour les jouer par cœur, sans partition, que pour l'ajusteur ; de même, la profession de chirurgien est, en partie, par les aptitudes pratiques qu'elle requiert (habileté, parfois force physique) une profession manuelle. Mais le statut de ces professions exclut de les considérer et de les classer comme telles.

Le qualificatif de manuel ne peut en effet être attribué à des activités considérées comme artistiques ou savantes. Sa définition est négative : « manuel » signifie " non intellectuel». Exercer un métier manuel, c'est réaliser, exécuter ce qui a été pensé par d'autres, conçu par des supérieurs ; " manuel » est spontanément réservé aux emplois qui occupent les positions subordonnées dans la hiérarchie sociale. Les métiers réputés les plus manuels sont les plus " matériels » et les plus " physiques ", ceux où l'on agit sur les choses et non sur les personnes, où l'on est en contact direct avec la matière inerte, ceux qui font le plus appel au corps, plus particulièrement aux muscles (levage, portage, frappe, etc.), et qui sont le plus directement exposés aux agressions et aux dangers du milieu naturel, les métiers des mines et les métiers de plein air, de chantier, plus que les métiers d'atelier. Les métiers

1. Cf. les analyses de B. Bernstein sur l'extra verbal channel (Bernstein, 1964). 
manuels sont aussi les métiers utiles, et donc utilitaires, par opposition aux activités et aux professions " désintéressées ", comme les arts, et aussi les sports, non moins physiques pourtant que les métiers du bâtiment et des travaux publics.

L'opposition entre manuel et non manuel est un des invariants caractéristiques du contexte culturel dans lequel le baccalauréat professionnel a été créé et s'est développé ; mais ce contexte a aussi été marqué par des changements sociaux susceptibles de modifier le rapport que les classes populaires entretiennent avec la culture dominante et avec l'école. Nous examinerons successivement ces deux points.

\section{1- Les changements}

La création, en 1985, du baccalauréat professionnel, constitue une promotion dans la hiérarchie scolaire de l'enseignement des métiers, jusque-là relégué au niveau de base de la hiérarchie des diplômes (le CAP équivalent du CEP)² ou à son niveau élémentaire (le BEP, Brevet d'études professionnelles, équivalent du Brevet) ; mais son succès ${ }^{3}$ est allé de pair avec la généralisation du baccalauréat (à laquelle il a contribué), et avec les conséquences de celle-ci : inflation et dévalorisation du diplôme, perte du prestige et du pouvoir de sélection que lui conférait sa rareté. L'obtention du baccalauréat cesse d'être une condition suffisante et devient de plus en plus une condition nécessaire pour accéder aux professions et aux positions supérieures, voire moyennes ; il faut être bachelier non pour monter, mais pour ne pas descendre dans la hiérarchie sociale. La dévalorisation des diplômes décourage les élèves issus des classes populaires ; elle contribue à leur désaffection croissante à l'égard des études, voire à leur rejet de l'école : dès lors que la réussite scolaire ne garantit plus la réussite sociale, pourquoi se priver, renoncer aux possibilités d'emploi et de promotion immédiates offertes par le milieu local et essayer de devenir un bon élève au risque de susciter l'hostilité du milieu, du groupe des pairs et des chefs de bande locaux ? Par ailleurs les réussites exceptionnelles, les plus sensationnelles, notamment dans les spectacles ou dans les sports, résultent souvent de parcours et de carrières extra-scolaires.

Les changements dans l'origine et la composition sociales des classes populaires transforment les cultures dont celles-ci sont porteuses, modifient le rapport qu'elles entretiennent avec la culture dominante, avec la culture savante transmise par l'école, et avec l'école ellemême. Le baccalauréat professionnel est créé alors que la part dans la population des catégories dont les élèves de l'enseignement professionnel sont le plus souvent issus décroît. C'est le cas des ouvriers et des artisans ${ }^{4}$. Ce déclin va de pair avec un exode agricole et un

2. Respectivement certificat d'aptitude professionnelle et certificat d'études primaires.

3. 2,8 \% de bacheliers professionnels dans une classe d'âge en 1990, 11,4 \% en 2000, 23,9\% en 2012. Repères et références statistiques sur les enseignements, la formation et la recherche, DEPP, septembre 2010. 2012 : http://cache.media.education.gouv.fr/file/2013/82/6/DEPP-NI-2013-02-resultats-definitifs-baccalaureat-session-2012_245826.pdf

4. $38,9 \%$ de la population active en $1962,32,6 \%$ en 1982, 25,6 \% en 1999, 23,1\% en 2007 pour les 
exode rural massifs ${ }^{5}$ qui modifient l'héritage culturel propre aux classes populaires : moins souvent enfants de paysans, les ouvriers sont moins souvent porteurs des savoir-faire et des dispositions liés aux métiers agricoles et au milieu rural.

L'urbanisation et l'immigration ont abouti au regroupement et à la relégation des couches les plus dominées, les plus pauvres et les moins intégrées des classes populaires dans des lieux spécifiques, les quartiers ou les banlieues dits "sensibles ${ }^{6}$. Cette mise à l'écart aboutit à la formation de sous-cultures fermées, à base locale, qui se définissent par la frontière qui les sépare des sous-cultures voisines et par leur distance par rapport à la culture standard perçue comme étrangère ${ }^{7}$. L'acculturation des indigènes de ces cultures bute sur l'obstacle de la langue, sur l'usage des vernaculaires, des parlers et des argots locaux ; pour les immigrés de première génération, le français est une langue étrangère, qu'il faut apprendre à l'école.

Autre facteur de marginalisation et d'exclusion, l'augmentation du chômage, auquel les ouvriers, et parmi eux les ouvriers non qualifiés et les manœuvres, sont les plus exposés ${ }^{8}$. L'anomie de fait qui en résulte va de pair avec la crise de l'idéal politique et social d'intégration hérité des Lumières et de la Révolution (" la République indivisible»). En rupture avec la conception ségrégationniste des états et des ordres héritée de l'Ancien Régime, cet idéal vise à ne laisser aucune classe à l'extérieur de la société, à intégrer socialement les classes populaires en les assimilant culturellement, par la diffusion des pratiques dominantes et par l'intermédiaire de l'école ${ }^{9}$. Il est de plus en plus concurrencé et contesté par une idéologie communautariste, fondée sur la reconnaissance et la valorisation (le " respect ") de toutes les cultures, et sur le rejet de l'acculturation à une culture commune standard ; alors que l'uniformisation linguistique, élément-clé de l'assimilation culturelle, était un des principaux objectifs de l'école républicaine, l'enseignement des langues régionales ou « minoritaires » est en passe de devenir obligatoire.

ouvriers, $11,8 \%$ des actifs en 1962, 8,5 \% en 1982, 5,9 \% en 2007 pour les artisans (regroupés avec les commerçants). Source : INSEE, Recensements de la population.

5. $16 \%$ d'agriculteurs en 1962, $2 \%$ en 2007. Source : Id.

6. Si la proportion des immigrés recensés dans la population active tend à rester stable $(6,4 \%$ en $1968,7,4 \%$ de 1975 à 1999, 7,9 \% en 2006), elle est beaucoup plus élevée dans certains quartiers des grandes villes ou des communes qui en sont proches (42,2\% à Aubervilliers, 36,6 \% à Saint-Denis, 24,8 \% à Vénissieux en 2012).

7. Sur le caractère local des cultures populaires et sur ses effets, $c f$. R. Hoggart (2013).

8. Le taux de chômage (population active, 15 ans et plus) passe de 12,4 \% en 1985 à 20,6 \% en 2013 pour les ouvriers non qualifiés, de $9,3 \%$ à $11,2 \%$ pour les ouvriers qualifiés, de $7,3 \%$ à $10 \%$ pour les employés et de 3,52 \% à 3,9\% pour les cadres et professions intellectuelles supérieures. Source INSEE, enquêtes Emploi. 9. Sur l'ambivalence de cet idéal, à la fois élitiste et égalitaire, et sur l'assimilation culturelle comme condition de l'intégration sociale, of. Grignon C., "La règle, la mode et le travail ", in Aymard M., Grignon C., Sabban F. (dir.), Le temps de manger, Alimentation, emploi du temps et rythmes sociaux, Paris, Ed. de la MSH, INRA, 1993. 


\section{2- Les invariants}

Dès lors que l'on change de perspective et d'échelle temporelle, qu'on se place dans le temps long de l'histoire, on observe au contraire des invariants qui se situent sans doute à un niveau plus fondamental. C'est le cas en particulier de la hiérarchie sociale des professions et des métiers, de la hiérarchie scolaire des ordres d'enseignement et de la correspondance entre ces hiérarchies.

L’enseignement des métiers continue d'occuper la position la plus basse dans la hiérarchie des ordres d'enseignement. On fait des études professionnelles par défaut, faute de mieux, c'est-à-dire faute d'avoir pu faire des études générales. Les écoles professionnelles, lycées compris, continuent d'être des instances de relégation, qui recrutent les meilleurs des moins bons élèves, une "élite de réprouvés" (Grignon, 1971). Lâge moyen à l'obtention du baccalauréat est nettement plus élevé pour les titulaires d'un baccalauréat professionnel que pour les titulaires d'un baccalauréat général ${ }^{10}$, ce qui ne s'explique pas seulement par la durée plus longue des études (quatre ans au lieu de trois jusqu’à la réforme de 2012) : $11 \%$ seulement des baccalauréats professionnels s'obtiennent "à l'heure " et $54 \%$ avec deux ans ou plus de retard, contre respectivement $63 \%$ et $12 \%$ des baccalauréats généraux (Education et Formation, 2001). En dépit des tentatives pour instaurer des "passerelles ", la circulation entre les ordres d'enseignement se fait à sens unique, de haut en bas ; quand on est passé par l'enseignement professionnel, on a peu de chances de repasser dans l'enseignement général : $6 \%$ seulement des titulaires d'un baccalauréat professionnel poursuivent des études à l'université, $16 \%$ préparent un BTS (brevet de technicien supérieur).

L'enseignement des métiers continue d'être évité par les classes dominantes et abandonné aux classes populaires. Alors que les enfants d'ouvriers sont proportionnellement deux fois moins nombreux que les enfants de cadres supérieurs ou de membres des professions libérales dans l'enseignement général (première et terminale des lycées), 15,3\% contre $30,4 \%$, ils sont cinq fois plus nombreux dans les classes préparant au baccalauréat professionnel, $35,5 \%$ contre $7,1 \%{ }^{11}$. Conséquence de la banalisation du baccalauréat, la facilitation de l'accès à l'enseignement supérieur a entrainé une dévalorisation des études universitaires les plus accessibles et un accroissement de la distance, tant académique que sociale, entre les niveaux de l'enseignement supérieur, entre son sommet, les Grandes Ecoles, et sa base, les STS. La dévalorisation du baccalauréat a entraîné une survalorisation des concours et des examens les plus sélectifs, les plus capables d'ériger des barrières entre les différents niveaux, et de sélectionner ainsi les « élites ".

L’enseignement professionnel se distingue de l'enseignement "général " par sa spécialisation. Nombreuses et diverses (environ 90), les spécialités du baccalauréat profes-

10. 21 ans et 5 mois contre 18 ans et 6 mois, Education et Formation, $\mathrm{n}^{\circ}$ 60, 2001.

11. A la rentrée 2013. Source : DEPP. 
sionnel s'ordonnent et se classent en fonction des savoirs auxquels elles correspondent, plutôt théoriques et matières à enseignement (le baccalauréat professionnel se rapprochant alors du baccalauréat technique), ou, à l'inverse, plutôt pratiques (la formation penchant du côté de l'apprentissage).

A cette hiérarchie scolaire se superpose une hiérarchie des spécialités en fonction des débouchés et des perspectives de carrière qu'elles offrent, et plus généralement de la position que les métiers auxquels elles préparent occupent dans la hiérarchie sociale. La plupart de ces métiers sont des métiers " manuels "; mais certaines de ces spécialités préparent à des métiers où l'on agit sur les personnes, et qui ne sont donc pas considérés comme manuels; c'est le cas par exemple des spécialités " accueil - relation clients et usagers", "gestion-administration " ou "services de proximité et vie locale ". Le statut des métiers du commerce est plus ambigu ; nombre d'entre eux (boulanger, poissonnier, boucher, «métiers du pressing et de la blanchisserie ») sont aussi des artisanats. Certains métiers de service, comme ceux auxquels prépare la spécialité " accompagnement soins et services à la personne ", peuvent inclure des tâches domestiques, matérielles et subordonnées.

En haut de la hiérarchie, à la frontière mais en deçà de la frontière qui sépare l'artisan de l'artiste, on trouve des "métiers d'art ", comme tailleur de pierres, tapissier ou facteur d'orgues. La restauration est partagée entre la haute cuisine, qui s'apparente au plus près à un art, et les basses besognes. A noter que les artisanats, et pas seulement les artisanats d'art, organisent, dans la tradition des corporations et du compagnonnage, des concours qui leur sont propres, couronnés par le titre de "meilleur ouvrier de France ". Intitulé paradoxal ; l'opposition entre métiers artisanaux et métiers ouvriers est en effet une opposition majeure à l'intérieur des métiers dits manuels. Symboles de la " qualité " et de la " tradition ", les métiers artisanaux offrent en outre des possibilités plus grandes de se mettre à son compte.

Lopposition entre les professions et les métiers, la dévalorisation des métiers manuels témoignent de la survivance d'une représentation et, pour une part, d'un état aristocratiques de la hiérarchie sociale. Dans cette perspective, les classes vraiment supérieures sont des classes oisives, servies par les classes laborieuses ; le travail est par définition servile, il n'existe pas de métiers, ni même de professions nobles. L'oisiveté va de pair avec l'ostentation : l'aristocratie n'a d'autre obligation que de paraître et de parader (Veblen, 1970).

Cette conception de la hiérarchie sociale est un archaïsme, lié à l'ancien régime et à la domination de la noblesse. Elle est pourtant loin de disparaitre. La permanence, voire le retour de la conception et de l'organisation aristocratiques de la société s'observent chaque fois que subsistent ou que s'introduisent dans les sociétés de classes des éléments caractéristiques et constitutifs des sociétés de castes, comme la séparation stricte entre les couches définies par leur position dans la hiérarchie sociale, l'impossibilité de passer de l'une à l'autre. On a vu que les examens et plus encore les concours intro- 
duisent des barrières entre les différents niveaux. Encore permettent-ils à ceux qu'ils sélectionnent de les franchir; ce n'est pas le cas lorsque l'appartenance à une strate va de pair avec la sélection par la naissance, qui est au principe même des castes.

Typiquement aristocratique, le critère de la naissance est incompatible avec l'esprit et la morale des sociétés de classes. On n'en assiste pas moins au retour en force, dans l'air du temps et dans l'opinion cultivée, de l'idéologie des origines et des « racines " $(\mathrm{y}$ compris dans sa variante populiste, les "quartiers de plèbe " se substituant aux "quartiers de noblesse ») (Grignon, 2013). L'origine est censée garantir l'authenticité et donc la qualité, qu'il s'agisse des produits (du terroir), ou des personnes (de qualité). La valorisation de l'authenticité renvoie à la notion d'identité, elle aussi de plus en plus en vogue, qui fait de la qualité l'émanation d'une essence.

\section{Bibliographie}

Bernstein B. (1964), «Elaborated and Restricted Codes: Their Social Origins and Some Consequences ", American Anthropologist, 66.

Grignon C. (1971), L'Ordre des choses, les fonctions sociales de l'enseignement technique, Paris, Ed. de Minuit.

Grignon C. (2013), "Présentation de 33 Newport Street " in Hoggart R., 33 Newport Street, Autobiographie d'un intellectuel issu des classes populaires anglaises, Paris, Ed. du Seuil, Coll. " Points Essais ».

Hoggart R. (2013), 33 Newport Street, Autobiographie d'un intellectuel issu des classes populaires anglaises, Paris, Ed. du Seuil, Coll. " Points Essais " [Gallimard Le Seuil, coll. " Hautes Etudes », 1991, trad. de A Local Habitation, Londres, Chatto and Windus, 1988].

Veblen Th. (1970), Théorie de la classe de loisir, Paris, Gallimard. 